\title{
Exploration of the associations of touch-screen tablet computer usage and musculoskeletal discomfort
}

\author{
Hsin-yu (Ariel) Chiang and Chien-Hsiou Liu* \\ Department of Occupational Therapy, College of Medicine, Fu Jen Catholic University, Taipei, Taiwan
}

Received 3 December 2014

Accepted 22 September 2015

\begin{abstract}
.
BACKGROUND: Tablet users may be at high risk of developing physical discomfort because of their usage behaviors and tablet design.

OBJECTIVE: Investigate the usage of tablets, variations in head and neck posture associated with different tablet tilt angles, and the association of tablet use with users' musculoskeletal discomfort.

METHODS: A survey of users' subjective perceptions conducted by questionnaire and measurements of users' postures by a 3D Motion analysis system was used to explore the effects of tablet use.

RESULTS: The questionnaire results indicated that over half of the participants reported physical discomfort after using tablets, with the most prevalent discomfort in the neck and shoulders, and more intensity of discomfort for the back although only few participants experienced it. Chi-squared tests indicated that significantly more participants who tended to use tablet computers to play games reported having musculoskeletal discomfort after using a tablet. In addition, preferences for tablet tilt angles varied across tasks (reading and game playing). The results from the 3D motion analysis revealed that head and neck flexion angles were significantly reduced when the tablets were positioned at relatively steep tilt angles. Neck flexion angle was significantly higher in game playing.

CONCLUSIONS: These data add information regarding to the usage of tablet and its associations with physical discomfort (significantly more participants who tended to use tablet computers to play games reported having musculoskeletal discomfort after using a tablet). Steep tilt angles (such as $60^{\circ}$ ) may cause tablet users to decrease their head and neck flexion angles, which could lead to a more neutral, effortless, and ergonomically correct posture. Maintaining proper neck posture during active activities such as game playing is recommended to avoid neck discomfort.
\end{abstract}

Keywords: Tablet tilt angle, physical discomfort, head and neck posture

\section{Introduction}

Mobile electronic devices with touch-screen features such as tablet computers (tablets) and smart phones are gaining popularity worldwide. Tablet

*Address for correspondence: Chien-Hsiou Liu, College of Medicine, Department of Occupational Therapy, No. 510, Zhongzheng Rd., Xinzhuang Dist., New Taipei City 24205, Taiwan. Tel.: +88602 29052091; Fax: +88602 2904 6743; E-mail: 070692@mail.fju.edu.tw. sales worldwide increased by $98 \%$ from the year 2011 (60 million) to the year 2012 (119 million) [1]. It is estimated that 201,825,000 tablets were sold worldwide in 2013, and it is expected that sales will reach 276 million in 2014. According to market projections, over 360 million tablets will be sold in 2016 [1]. Despite the rapid growth in the popularity of touchscreen tablets, only a few studies have been done to establish ergonomic guidelines for their usability [2-4]. There is a clear and present need for ergonomic 
evaluations of tablets so that recommendations can be formulated to reduce the possibility of potential musculoskeletal discomfort or injuries in the users and increase the usability of the tablets $[2,3]$.

According to Chung's investigation of tablet usage, the main use is for entertainment, such as web browsing, game playing, and video clip watching [5]. Related studies have investigated how the positions of desktop computer monitors and laptop computers affect neck and shoulder posture. According to one study, a higher screen height setting is positively correlated with a more erect neck angle (mean coefficient of correlation $=0.94$ ), and EMG activity in the neck muscles is related to neck angle [6]. As a result, higher display locations (e.g., the top of the screen set at the participant's eye height) generally leads to neutral positions of the neck and head, while lower display locations (e.g., the screen set horizontally on the desk) leads to increasing flexed postures of the head and neck and is hypothesized to be a risky position, one that may cause heavy loads and strain on the muscles and thereby put users at risk of developing musculoskeletal discomfort or pain $[3,7,8]$.

Although the height of the display location and its association with muscle strain/discomfort has been studied extensively, research to develop usage guidelines to prevent musculoskeletal discomfort due to tablet use is still limited. Tablets provide an integrated interface that combines touch and display in one screen, and this design may elicit usage behaviors unlike those common to PC or laptop computer users. For example, users may adopt a floating forearm posture so that they do not inadvertently activate the touch screen, a posture that can lead to discomfort in the neck and shoulder regions [9]. In addition, due to the sudden popularity of the tablets, suitable ergonomic parameters for posture and usage guidelines, such as the effects of preferred tablet tilt angles, have not been developed comprehensively yet. Young et al. [3] reported that head and neck angles during tablet use were greater than those previously reported for desktop and laptop computing, especially when the tablet was placed on the lap or positioned at shallower tilt angles $\left(15^{\circ}\right.$ and $\left.45^{\circ}\right)$. Postural differences (head and neck angles) between tablets also tend to be driven by case designs, which provide significantly different tilt angles (e.g., $15^{\circ}, 45^{\circ}$, and $63^{\circ}$ ). Young et al. [3] reported that head and neck flexion was significantly reduced (to within the neutral range) when two different tablet computers were placed on a table and positioned at relatively steep tilt angles $\left(63^{\circ}\right)$.
Therefore, this configuration (tablet placed on table and positioned at different tilt angles) was used in the present study to test the effects of tablet tilt angles on head and neck angles in a relatively large sample.

Tablets can be tilted during use [4]. Young et al. [3] reported that the preferred tablet tilt angle changes depending on the location of the tablet. According to Young et al. [3], when users can control the tilt angle (tablet in hand-held, placed on lap position), they select an average tilt angle of approximately $36^{\circ}$ on average, and McLoon and Albin found when the tablet is placed on a table [4], most users find a tablet tilt angle of $34^{\circ}$ most preferable on average. However, the small sample size (9-15 participants) used in both studies might diminish the statistical power, and further studies with larger sample sizes may better define tilt angle preferences.

Tablet users may be at high risk of developing neck discomfort due to their current behaviors and tablet design $[3,8]$. However, the context of touchscreen tablet usage, such as preferred case-supported tilt angle, preferred posture, and preferred task, and the associations between usage and users' musculoskeletal discomfort have not been fully explored to establish ergonomic guidelines for their usability. In addition, it is still unclear how the tablet tilt angle affects head and neck flexion angles and subjective experience since this type of usage has been and continues to be associated with discomfort and pain in the neck and shoulders [10]. This study was designed to collect additional data in this space using both subjective and objective methodologies. In particular, a self-developed questionnaire and a 3D motion analysis system, were applied to investigate the following: 1) variables (time spent, posture, and task performed on tablet) that may cause musculoskeletal discomfort related to touch-screen tablet usage; and 2) variations in head and neck posture at different tablet tilt angles.

\section{Methods and results}

\subsection{Experiment $1-$ Survey and seated tablet tilt preference}

\subsubsection{Participants}

For Experiment 1, 80 college students (26 males and 54 female) who were over the age of 20 and had used tablets for over 3 months were recruited. Of the 80 participants, 75 were right-handed. None reported having any musculoskeletal disorders. 


\subsubsection{Questionnaire "habits of tablet usage and user discomfort"}

To develop the questionnaire for this study, the researcher conducted a review of the related literature and interviewed tablet computer users to identify the most relevant issues. For a test of the content validity, the trial version was piloted with 25 college students who used tablet computers and three college professors with backgrounds in ergonomics and psychometrics, from whom feedback was collected and used as a reference to improve the content and wording of the questionnaire. The final version of the questionnaire, "Habits of Tablet Usage and User Discomfort, contained six sections: 1) personal information: this section contains questions such as age, gender, dominant hand and previous injuries; 2) commonly used tablet tilt angles for different tasks: all of the common tasks performed on tablet (chosen from the feedbacks from the 25 college students and professors with the background in ergonomics) are listed on the questionnaire (see Table 1 for the list of different kinds of tasks) and the research assistant measured the tablet tilt angle under different tasks; 3) time spent on activities other than using a tablet computer: Questions regarding time spent (under $1 \mathrm{hr}, 1-2 \mathrm{hrs}, 2-3 \mathrm{hrs}, 3-4 \mathrm{hrs}, 4-5 \mathrm{hrs}$ and above $5 \mathrm{hrs}$ ) on activities other than using a tablet computer in an average day, such as time spent on reading, using computer and smart phone are asked to rule out heavy smart phone or computer users since these habits might cause musculoskeletal discomforts as well; 4) time spent using tablet computers in different contexts; 5) questions regarding the influence of the use of tablet computers, such whether the participants experienced any musculoskeletal discomfort (if yes, the participant was asked to color in the body part(s) on the questionnaire); and 6) a visual analogue scale (VAS) of bodily pain induced by tablet use [11]. The anchors depict extreme states ranging from "no pain" to "worst pain imaginable" with the length of $10 \mathrm{~cm}$.

\subsubsection{Procedure}

This first phase of the study was conducted in the form of a questionnaire survey. Possible participants who either owned or had experience with using tablet computers were informed that participation was voluntary and anonymous. Informed consent was obtained before the participants filled out the questionnaires. Participants generally took about 10 minutes to complete the questionnaire in an ergonomic laboratory. The preferred tablet tilt angles were measured using a goniometer with the tablet (a 4th generation iPad) placed on a table (height $=75.3 \mathrm{~cm}$ ) and the participant seated in a chair (seat pan height $=44.7 \mathrm{~cm}$ ) (Fig. 1). In order to investigate the preferred tablet tilt angle, the height of the table and the chair used in this study conformed to the standard size of the furniture in Taiwan (table height: $73-76 \mathrm{~cm}$; chair height: $40-50 \mathrm{~cm}$ ) to simulate a commonly seen context of tablet using [12]. The heights of the chair and the table were not adjustable. The tablet was positioned horizontally on the table to the user. In Section 2 of the questionnaire, a list of the common tasks performed on tablet was provided. If the participants did use tablet for those tasks, they would have to set up the tablet in that angle for each task so the research assistant could measure the tilt angle of the tablet. The tilt angle was adjusted by the tablet cases (brought by the participants themselves). Figure 1 shows the setup of Experiment 1. After the completion of the survey, gratuities were presented to the participants.

Table 1

Tablet tilt angle under different tasks performed on tablet computers

\begin{tabular}{clccr}
\hline & & $\%^{*}$ & $\begin{array}{c}\text { Mean tablet tilt angle } \\
\text { (in degrees) }\end{array}$ & SD \\
\hline Passive & Web browsing & 97.2 & 37 & 19 \\
activities & Social networking & 97.2 & 40 & 20 \\
& Movie watching & 93.1 & 53 & 23 \\
& Photos (browsing) & 86.3 & 40 & 20 \\
& Music (listening) & 49.3 & 4 & 13 \\
Active & Reading & 56.1 & 37 & 21 \\
activities & E-mail & 78.0 & 31 & 21 \\
& Texting & 73.9 & 33 & 24 \\
& Game playing & 83.5 & 25 & 23 \\
& Word processing & 31.5 & 33 & 39 \\
\hline
\end{tabular}

*Note: \% indicates percentage of the participants from experiment 1. 


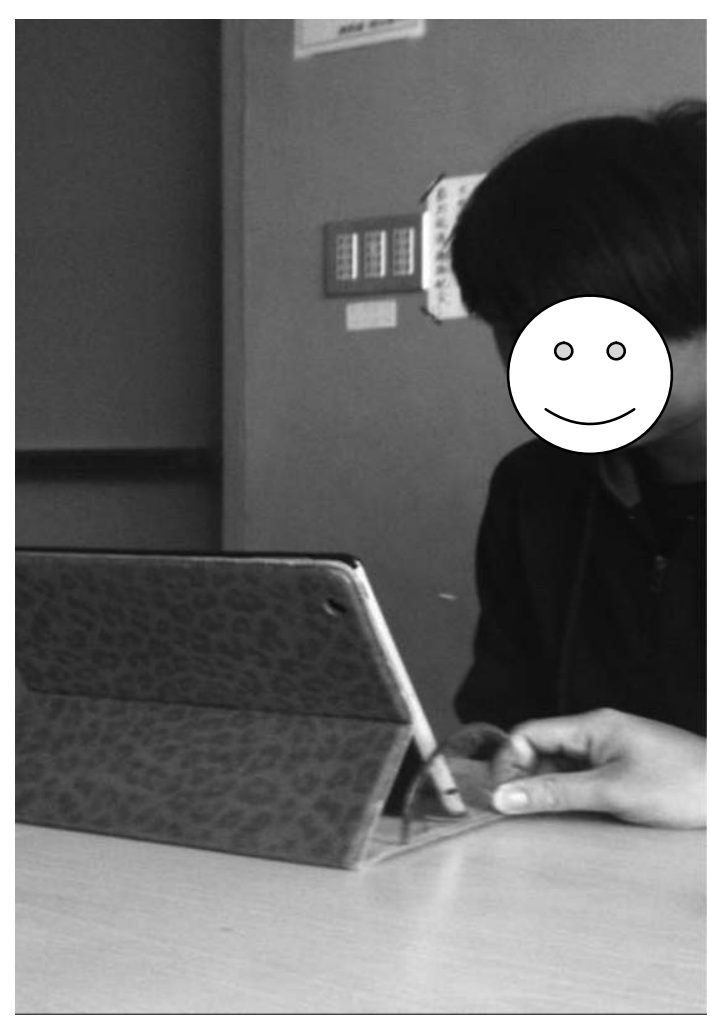

Fig. 1. Setup in Experiment 1.

\subsubsection{Data analysis}

SPSS 19.0 was used to analyze the data in this study. Descriptive analyses (means, standard deviation, and percentages of responses for each item), Chi-square, and independent $t$-test were applied. The $\alpha$ value of this study is 0.05 .

\subsubsection{Results}

Table 2 presents the number of participants who reported musculoskeletal discomfort after tablet usage in different body regions on the questionnaire. Of the 80 participants, 44 reported having discomfort after using tablet computers. The Chi-squared test showed no significant differences between discomfort and gender $(p=0.27)$, age $(p=0.051)$, and handedness $(p=0.104)$. Neck and shoulders are the most frequent areas of pain reported by our participants. Thirty of the participants reported neck pain after tablet use and over 20 participants claimed pain in shoulders. The participants also reported that the most intense discomfort was in the back (VAS $=5.6 \mathrm{~cm}$ ) although only six participants experienced it.
Table 2

VAS scores from different body regions $(N=80)$

\begin{tabular}{lcc}
\hline Body regions & $\begin{array}{c}\text { Numbers of participants } \\
\text { claimed to have pain related } \\
\text { to tablet usage }\end{array}$ & $\begin{array}{c}\text { Mean VAS } \\
\text { scores (SD) }\end{array}$ \\
\hline Neck & $30(37.5 \%)$ & $4.91(2.353)$ \\
Right shoulder & $24(30 \%)$ & $4.98(2.506)$ \\
Left shoulder & $21(26.3 \%)$ & $5.26(2.388)$ \\
Waist & $11(13.75 \%)$ & $4.40(2.535)$ \\
Right wrist & $13(16.3 \%)$ & $4.08(1.881)$ \\
Right forearm & $6(7.5 \%)$ & $3.17(1.155)$ \\
Right upper arm & $4(5 \%)$ & $2.95(1.748)$ \\
Left wrist & $5(6.3 \%)$ & $4.88(1.894)$ \\
Left forearm & $2(2.5 \%)$ & $5.25(2.900)$ \\
Left upper arm & $1(1.3 \%)$ & $0.70(\mathrm{~N} / \mathrm{A})$ \\
Back & $6(7.5 \%)$ & $5.60(1.490)$ \\
\hline
\end{tabular}

As for daily tablet usage, 28 (35\%) of the participants reported usage times of 2 to 3 hours per day. Twenty-two people (27.5\%) reported that they used tablets 1 to 2 hours per day, and 13 (16.3\%) reported times of 3 to 4 hours per day. Only nine of the participants $(11.3 \%)$ used their tablets for 4 to 5 hours per day, and four (5\%) reported spending 5 to 6 hours on their tablets per day. However, the Chi-squared test found no significant difference between time spent using tablet computers and musculoskeletal discomfort after tablet use $(p=0.106)$. In addition, the Chi-squared test indicated that the discomfort had no relation to the time spent on reading per day $(p=0.708)$, time spent on a smart phone $(p=0.272)$, time spent on a computer $(p=0.345)$, or any other activities $(p=0.110)$.

Preferences for tablet tilt angle may vary across tasks. Information on the preferred angles for different tasks is presented in Table 1. In addition, the mean of participants' preferred tilt angles of the tablet of passive tasks (Social networking, web browsing, movie watching, photos (browsing), music (listening) and reading $)$ was $33^{\circ}(\mathrm{SD}=12$, median $=31)$; the preferred angle for active tasks (E-mail, texting, game playing and word processing) was $37^{\circ}(\mathrm{SD}=14$, median $=35$ ). Our participants had used their tablets for an average of 13.684 months $(S D=10.426)$. Information related to the various tasks performed on the tablet computers and the percentages of participants who used tablets to perform specific tasks are listed in Table 1. Most of the tablet users (97.2\%) utilized tablets for socializing with their friends via Facebook $^{\mathrm{TM}}$ and browsing websites.

As for the relationship between different activities and musculoskeletal discomfort after using a tablet, the Chi-squared tests indicated that significantly more participants who tended to use tablet 
computers to play games reported having musculoskeletal discomfort after using a tablet $(p=0.017)$. However, no significant relationship was found between participants' musculoskeletal discomfort after using a tablet and different tasks performed on the tablet such as social networking $(p=0.205)$, web browsing $(p=0.859)$, movie watching $(p=0.858)$, photos (browsing) $(p=0.343)$, music (listening) $(p=0.565)$, reading $(p=0.335)$, E-mail $(p=0.563)$, texting $(p=0.369)$, and word processing $(p=0.641)$.

\subsection{Experiment 2}

\subsubsection{Participants}

For Experiment 2, 30 college students over the age of 20 who were familiar with the use of tablet computers and had no current or previous history of musculoskeletal disorders of the head, neck, back, or upper extremities were recruited. The heights of the participants ranged from 160 to $170 \mathrm{~cm}$. Four males and 26 females were recruited.

\subsubsection{Instrument and materials}

2.2.2.1. Tablet. The tablet computer used in this study was the iPad (4th generation), with a height of $241.2 \mathrm{~mm}$, width of $185.7 \mathrm{~mm}$, and depth of $9.4 \mathrm{~mm}$. The mass of it is $652 \mathrm{~g}$. The tablet was running the $\mathrm{iOS}$ 7.0 operating system. The iPad 4 th generation (same as the iPad air) has a display diagonal of $246.4 \mathrm{~mm}$. All trials were conducted with the tablet in landscape orientation, held in place by a case that could be adjusted to tilt the tablet computer (Fig. 2). The tablet was placed on a fixed spot on the table. The horizontal distance between the participant and the tablet was $30 \mathrm{~cm}$ (measured from the mid-point of the iPad to the user's upper trunk).

\subsubsection{3D Motion analysis system. The primary} outcomes were head and neck postures presented by head and neck flexion angles (Fig. 3). The head flexion and neck flexion angles were measured using a 3D motion analysis system (Optotrak Certus). Two clusters of four infrared light emitting diodes (IREDs) fixed to a rigid surface were secured to the head and upper trunk (Fig. 4). The positions of these IREDs were tracked in three dimensions at $100 \mathrm{~Hz}$, recorded on a personal computer, and digitally filtered through a filter with a $5 \mathrm{~Hz}$ cutoff frequency. Using the system's digitizing probe, the locations of the bilateral tragic, the $\mathrm{C} 7$ spinous process, and right and left acromion boney landmarks were digitized relative to their associated IRED clusters. In order to calculate

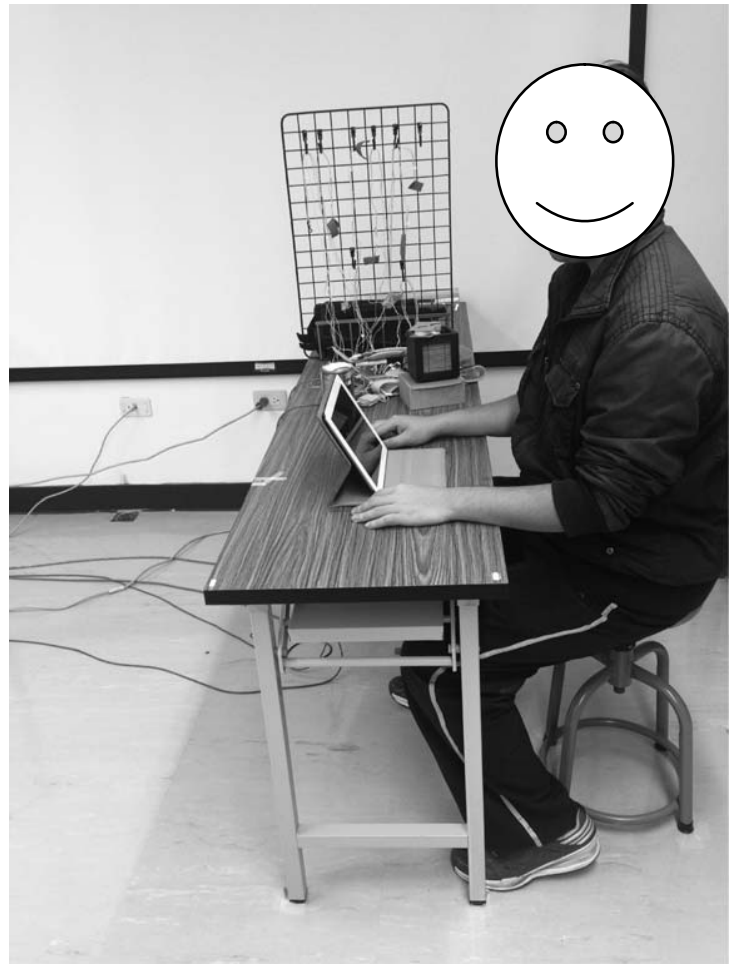

Fig. 2. Setup in Experiment 2.

the head and neck flexion angles, additional 3D locations were specified as follows: midpoint between the left and right tragi (representing the occiput-cervical joint "OC") (Fig. 3). For the duration of measurements, the position and orientation of each landmark were calculated based on the location of their associated IRED clusters [17]. Angles from the landmark positions are presented in Fig. 3.

\subsubsection{Procedure}

A laboratory-based repeated measures experiment was conducted to test whether the tablet tilt angle affected the head and neck posture of the user. Experienced tablet computer users completed a set of simulated tasks on a tablet computer at three different tilt angles $\left(0^{\circ}, 45^{\circ}\right.$, and $\left.60^{\circ}\right)$. Each participant gave informed consent before the beginning of the study. Participants performed all the tasks while seated in a chair with a seat pan height of $44.7 \mathrm{~cm}$, a backrest, and no arm rests. The tablet computer was positioned on a table with a height of $75.3 \mathrm{~cm}$, based on a previous study suggesting that most users preferred to use tablet computers at a table for most tasks [13]. In addition, the chair, desk, and tablet were all positioned to minimize glare on the tablet screen. 


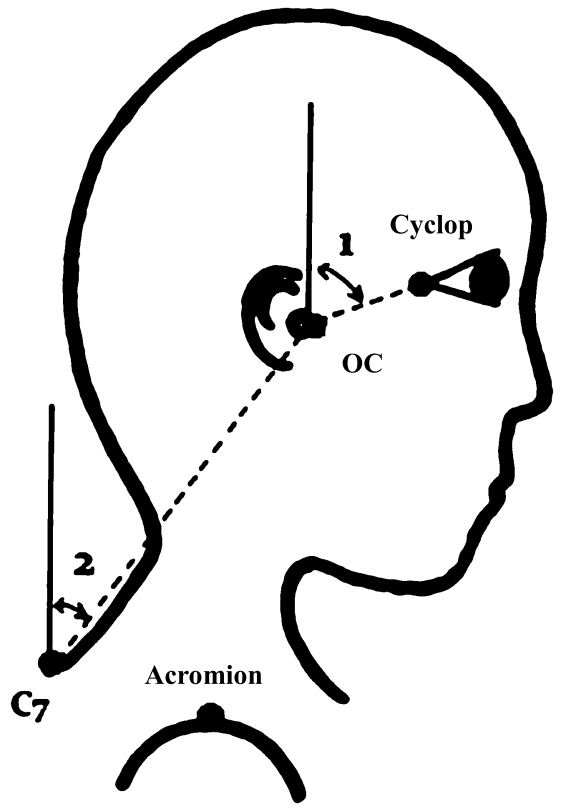

Fig. 3. Head and neck flexion angles. Head flexion angle (1) is the angle between the global vertical and the vector pointing from occiput-cervical (OC) to Cyclops (midpoint between the left and right outer canthi), Neck flexion angle (2) is the angle between global vertical and vector pointing from $\mathrm{C} 7$ to $\mathrm{OC}$.

All of the participants were asked to perform two different tasks (playing a game, Candy Shoot HD, and reading a news website, Next Media Interactive Limited). They performed each task for five minutes in randomized order, with 2-minute breaks after each task, under three different tablet tilt angle conditions in randomized order as well. The two tasks are the representatives of two typical tablet usagesThe Candy Shoot HD (playing a game), and Next Media Interactive Limited (reading a news website). The game playing task represents a common active shooting game that is available on most computers. The reading task (passive activity) consisted of navigating through pages and reading online newspaper articles. The tablet computer was placed directly in front of the participant on a predetermined spot on the table.

When performing each task, mean values from measures of the head and neck angles were taken every $1 / 100$ second in the middle of each experimental condition for a total 30 seconds; that is, the angles were taken at the 2.5-minute time point during each 5-minute game/reading task. After the completion of the survey, gratuities were presented to the participants.

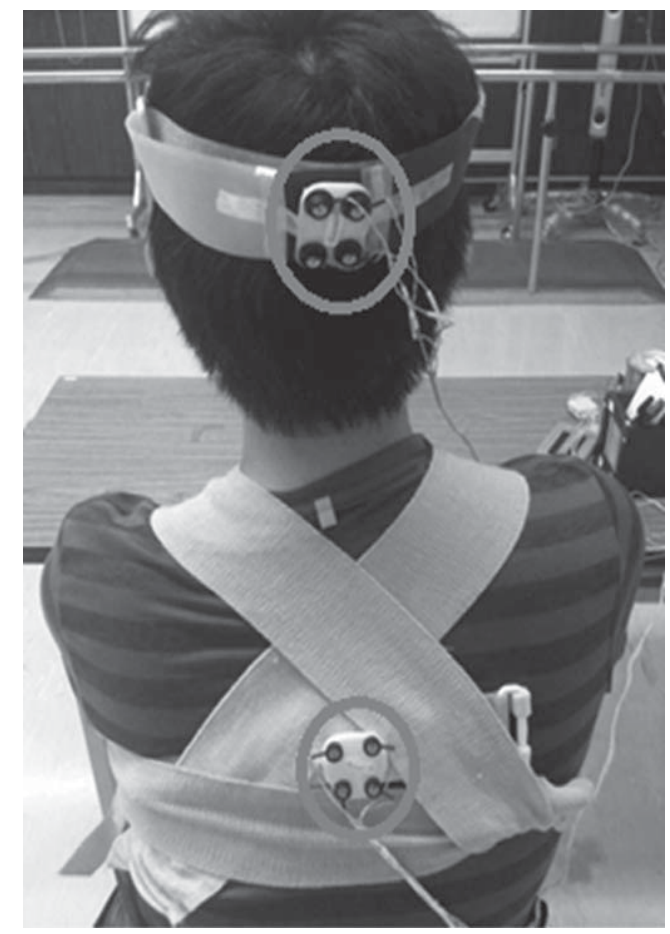

Fig. 4. Two clusters of four infrared light emitting diodes (IREDs) fixed to a rigid surface secured to the participant's head and upper trunk.

\subsubsection{Data analysis}

SPSS 19.0 was used to analyze the data in this study. In order to test the hypothesis that head and neck angles varied across different tablet tilt angles, repeated measure analysis of variance for two postures (head and neck angles) along with three tablet tilt angles were applied. Post-hoc LSD test (least significant difference) was used to determined if differences in postures existed between different tablet tilt angles. The $\alpha$ value of this study is 0.05 .

\subsubsection{Results}

ANOVAs were conducted to identify the relationships between different tablet tilt angles and neck and head flexion angles. For the head flexion angle, no significant interaction was found between tablet tilt angle and different tasks (playing a game and reading $)\left(\mathrm{F}_{2,58}=1.229, p=0.277\right)$. A significant main effect was observed for different tablet tilt angles $\left(\mathrm{F}_{2,58}=70.627, p<0.001\right)$. Significant differences were found between tablet tilt angles and head $(p<0.001)$ flexion angles by post-hoc LSD test, which indicated that head flexion was significantly reduced when the tablet computers wee positioned at relatively steeper tilt angles $\left(45^{\circ}\right.$ and $\left.60^{\circ}\right)$ and when 
the height of the screen changed with the tablet tilt angle. A post-hoc LSD test also showed that the head flexion angle at $0^{\circ}$ tilt angle $(115.224 \pm 1.684)$ was significantly greater than the head flexion angle at $45^{\circ}(103.941 \pm 1.830)(p<0.001)$, and that the head flexion angle at $45^{\circ}$ tilt angle $(103.941 \pm 1.830)$ was significantly greater than the head flexion angle at $60^{\circ}$ $(98.050 \pm 1.362)(p<0.001)$ (Fig. 5). No main effect of different tasks was found for head flexion angle $\left(\mathrm{F}_{1,29}=1.229, p=0.277\right)$.

For the neck flexion angle, no significant interaction was found between tablet tilt angle and different tasks (playing a game and reading) $(\mathrm{F} 2,58=0.838$, $p=0.438$ ). A post-hoc LSD test indicated that the neck flexion angle at $0^{\circ}$ tilt angle $(79.671 \pm 1.796)$ was significantly greater than the neck flexion angle at $45^{\circ}(71.4061 \pm 2.255)(p<0.001)$, and that the neck flexion angle at $45^{\circ}$ tilt angle $(71.4061 \pm 2.255)$ was significantly greater than the neck flexion angle at $60^{\circ}(64.298 \pm 1.989)(p<0.001)$. A significant main effect was observed for different tasks (playing a game and reading), which indicated that the neck flexion angle was significantly higher $(p=0.036)$ in

(a)

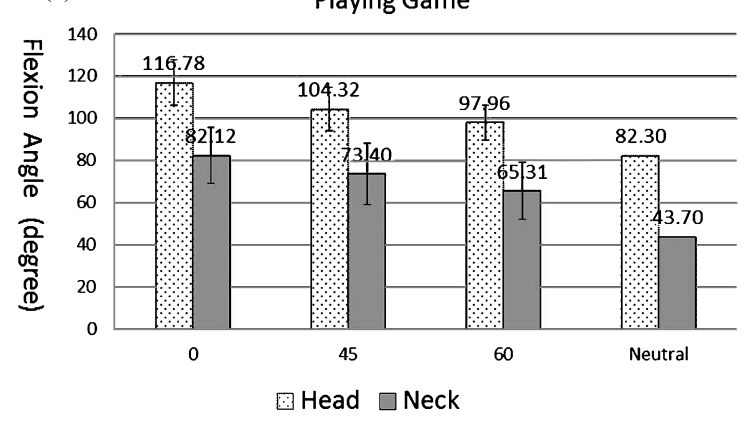

(b)

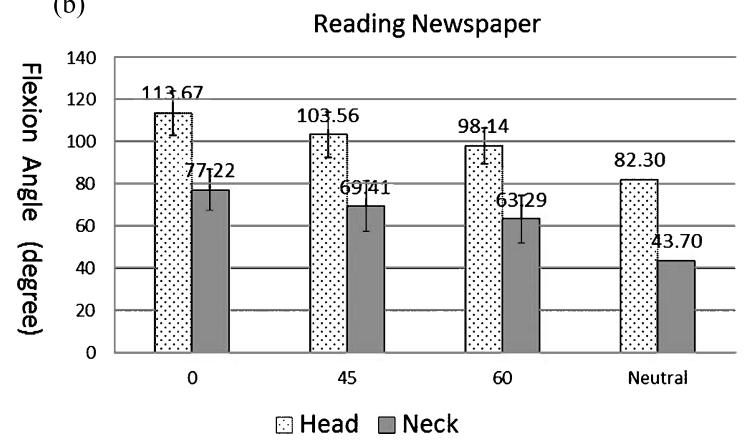

Fig. 5. Head and neck flexion angle in (a) Playing Game (game playing task) and (b) Reading Newspaper (reading task). *Note: "Neutral" is a self-selected angle, which means the most comfortable head flexion and neck flexion angle when subjects sit at their greatest height [14]. the game playing task $(73.608 \pm 2.201)$ than in the reading task (69.975 \pm 1.646$)$ (Fig. 5) [14].

\section{Discussion}

In the current study, a questionnaire, "Habits of Tablet Usage and User Discomfort", and objective experimental data from a 3D Motion analysis system were used to explore the effects of tablet computer use. Consistent with previous findings [3, 4], the results from this study revealed that head and neck flexion were significantly reduced when the tablet computers were positioned at relatively steeper tilt angles $\left(45^{\circ}\right.$ and $\left.60^{\circ}\right)$. According to the results of experiment 2 , when compared with $0^{\circ}$ and $45^{\circ}$, the neck and head flexion angles were closer to the neutral positions (most comfortable postures of head and neck) of head and neck when the tablet was in $60^{\circ}$, both in reading newspaper and game playing tasks (Fig. 5). Therefore, the tablet tilt angle of $60^{\circ}$ is recommended. Villanueva et al. [6] reported that neck extensor muscle activity decreased significantly when the users' necks were more vertical, and that this upright neck position would considerably reduce the load on the neck. However, regarding the users' perceptions recorded on a questionnaire, extreme tilt angles, were rated as uncomfortable for the arm and hands, according to a study by Albin and McLoone. Further study with longer task performance times and slightly lower tablet tilt angles (such as $35^{\circ}$ and $45^{\circ}$ ) is recommended to find the appropriate balance between neck flexion and hand and arm comfort.

Our results suggest that a steep tilt angle may cause tablet computer users to decrease their head and neck flexion angles, therefore leading to a more neutral, effortless, and ergonomically correct posture. Yen's study supports these findings. There, the fatigue level of the neck, back, and waist reached the highest point when the tablet was placed at $0^{\circ}$. Therefore, we propose that tablet computer users avoid placing their tablets flat on the table $\left(0^{\circ}\right)$ to decrease the load on the neck muscles. Additionally, the use of tablet cases or stands to create a steep viewing angle is suggested [2]. Finally, our results demonstrated no significant differences between the head flexion angles of two different tasks (reading and game playing). Further studies examining the effects of various tablet tasks and tilt angles on the positions of other body parts are needed to clarify the interrelationship.

Our results indicated that the mean of participants' preferred tablet tilt angles was between $33^{\circ}-36^{\circ}$, 
which is very close to the most preferable self-chosen tile angles $\left(34^{\circ}\right.$ and $\left.36^{\circ}\right)$ in previous studies [3, 4]. Similar to the Albin and McLoone's study [4], measure of the tilt angle was taken when the tablet was placed on the table in this study. When compared to Young and colleagues' study [3], the measure was taken on the lap. In Young and colleagues' study, they suggested preferred tilt angle most likely changes depending on location of the tablet angle and corresponds to tilt angles that allows for near perpendicular viewing angles, which optimizes luminance and reduces perspective distortion of the display. Our results also correspond to the finding of a previous study that preferences for tablet tilt angles may vary across tasks [13].

Interestingly, in Toy et al.'s study, they verified that people preferred to have the tablet flat on the desk when performing gaming and drawing tasks, whereas our participants preferred an angle of $25.25^{\circ}$ while gaming. This difference might be due to the different natures of the games. For example, when much tapping is required, the users may prefer to lay the tablet flat on the table to prevent sudden shifts in the position of the tablet due to heavy tapping.

As for the reading task, our experiment 2 revealed that the neck and head flexion angles were closer to the neutral positions of head and neck when the tablet was placed in $60^{\circ}$ in the reading task. Consistently with the previous studies $[15,16]$, placing a tablet flat on the table (at $0^{\circ}$ ) resulted in significantly greater mechanical demand on the neck muscles than placing tablet in a steep viewing angle $\left(73^{\circ}\right)$. In addition, placing the tablet at $0^{\circ}$ on the table for reading caused significantly more flexion of head and neck and three times more mechanical demand on neck muscles compared to its neutral posture [16]. As a result, placing tablet at $0^{\circ}$ on the table is not recommended for reading.

Our results from experiment 2 indicated different tasks (reading and game playing) significantly affect the neck flexion angle (neck flexion angle was significantly higher in the active activity - game playing task). Additionally, from experiment 1 , a high percantage of our participants used the tablet to play games. Therefore, maintaining proper neck posture during active activities such as game playing is recommended to avoid neck discomfort.

Our statistical analysis found no significant difference between various tasks performed on tablet computers (except game playing) and musculoskeletal discomfort. The reasons might be as follows: 1) The participants answered the questionnaire accord- ing to their overall impressions, so there may have been some inaccurate memories or impressions; 2) when the users sensed discomfort, they might have changed the tablet tilt angle or/and posture to alleviate the discomfort; and 3) other unexamined factors, such as users' physical and psychological contexts and the applications being used, might affect users' postures and therefore reduce discomfort. Further studies examing the effects of other variables, such as users' physical and psychological contexts, applications, and user discomfort, are needed to clarify the interrelationship.

From the questionnaire "Habits of Tablet Usage and User Discomfort", we discovered that participants were more likely to experience moderate pain in the neck (30 people) and shoulder region (24 people reported pain in the right shoulder and 21 for left shoulder) after using tablet computers. To reduce such physical discomfort, it is strongly recommended that users' head and neck posture be improved by adjusting the tablet tilt angle to decrease the neck muscle load through the use of a tablet case which allows the user to adjust the tablet to appropriate angles.

\subsection{Limitations}

The results of this study need to be interpreted with caution. First, the participants may have changed how they normally interacted with their tablets, since the experiment was conducted in a laboratory with simulated tasks and instruments attached to them. Although the questionnaire "Habits of Tablet Usage and User Discomfort" was applied to investigate users' habits, such as preferred angles, time spent per day, posture, and tasks performed on a tablet, selfreport data from memory may not always be accurate.

\section{Conclusion}

The results of this study revealed that head and neck flexion angles are significantly reduced when the tablet computers are positioned at relatively steeper tilt angles (such as $60^{\circ}$ ). A steep tilt angle may help users to decrease their head and neck flexion angles, thereby leading to a more neutral, effortless, and ergonomically correct posture. These data suggest that head and neck posture can be improved by adjusting the tablet tilt angle through the use of a tablet case which allows the user to adjust the tablet to appropriate angles. 
Based on the reports from participants, the use of tablet computers is associated with pain in the neck (30 participants), left shoulder (21 participants), and right shoulder (24 participants). In addition, Chi-squared tests indicated that significantly more participants who tended to use tablet computers to play games reported having musculoskeletal discomfort after using a tablet. Different tasks (reading and game playing task) significantly affect the neck flexion angle (neck flexion angle was significantly higher in the active activity - game playing task). Therefore, maintaining proper neck posture during active activities such as game playing is recommended to avoid neck discomfort.

\section{References}

[1] Gartner I. Gartner says worldwide media tablet sales to reach 119 million units in 2012 In: Stamford C, editor. 2012.

[2] Young JG, Trudeau MB, Odell D, Marinelli K, Dennerlein JT. Wrist and shoulder posture and muscle activities during touch-screen tablet use: Effects of usage configuration, tablet type, and interacting hand. Work 2013;45(1): 59-71.

[3] Young JG, Trudeau M, Odell D, Marinelli K, Dennerlein JT. Touch-screen tablet user configurations and case-supported tilt affect head and neck flexion angles. Work 2012;41: 81-91.

[4] Albin TJ, McLoone HE. The effect of tablet tilt angle on users' preference, postures, and performance. Work 2013.

[5] Yu-Hsun C. Activity-centered design-Explore the context of use of tablet PC. Taipei: National Taipei Technology University; 2012

[6] Villanueva MBG, Jonai H, Sotoyama M, Hisanaga N, Takeuchi Y, Saito S. Sitting posture and neck and shoulder muscle activities at different screen height setting of the visual display terminal. Industrial Health 1997;35(3):330-6.

[7] Straker L, Pollock C, Burgess-Limerick R, Skoss R, Coleman J. The impact of computer display height and desk design on muscle activity during information technology work by young adults. Journal of Electromyography and Kinesiology 2008;18(4):606-17.

[8] Straker L, Skoss R, Burnett A, Burgess-Limerick R. Effect of visual display height on modelled upper and lower cervical gravitational moment, muscle capacity and relative strain. Ergonomics 2009;52(2):204-21.

[9] Shin G, Zhu X, editors. Ergonomic issues associated with the use of touchscreen desktop PC. Human Factors and Ergonomics society 55th Annual Meeting; 2011.

[10] Siu DC, Tse LA, Yu IT, Griffiths SM. Computer products usage and prevalence of computer related musculoskeletal discomfort among adolescents. Work 2009;34(4): 449-54.

[11] Williamson A, Hoggart B. Pain: A review of three commonly used pain rating scales. Journal of Clinical Nursing 2005;14(7):798-804.

[12] Sstandard size for the commonly seen furniture 2010 [cited 2015 Mar, 8]

[13] Toy KJ, Peres SC, David TY, Nery A, Phillips RG, editors. Examining User Preference in Interacting with Touchscreen Devices. Proceedings of the Human Factors and Ergonomics Society 56th Annual Meeting; 2012.

[14] Ankrum DR, Nemeth KJ, editors. Head and Neck Posture at Computer Workstations - What's Neutral? Proceedings of the Human Factors and Ergonomics Society Annual Meeting, 2000.

[15] Nevins D, Monda S, Lin D, Vasavada A, editors. Mechanical demand of forward head postures associated with tablet PC use. American Society of Biomechanics Northwest Regional Meeting; 2013; University of Idaho, Moscow, ID.

[16] Vasavada AN, Nevins DD, Monda SM, Hughes E, Lin DC. Gravitational demand on the neck musculature during tablet computer use. Ergonomics 2015;58(6):990-1004.

[17] Yen C-C. A survey of physical fatigue during use of a tablet LCD monitor. Journal of Chung Cheng Institute of Technology 2011;40(1):49-61. 